\section{Aids e envelhecimento: \\ características dos casos com idade igual ou maior que 50 anos em Pernambuco, de 1990 a 2000}

\section{Aids and aging: characteristics of cases aged 50 years or more in the State of Pernambuco, from 1999 to 2000}

Fábia Alexandra Pottes'

Ana Maria de Brito²

Giselle Campozana Gouveia ${ }^{2}$

Ednaldo Cavalcante de Araújo'

Rosa Maria Carneiro ${ }^{3}$

'Departamento de Enfermagem da Universidade Federal de Pernambuco UFPE

${ }^{2}$ Departamento de Saúde Coletiva-NESC, Centro de Pesquisa Aggeu Magalhães, Fundação Oswaldo Cruz.

${ }^{3}$ Departamento de Medicina Social da Universidade Federal de Pernambuco UFPE

Correspondência: Fábia Alexandra Pottes. Av. Moraes Rego, 1235 - $1^{\circ}$ andar, Bloco A do Hospital das Clinicas - Cidade Universitária - Recife, PE CEP 50.670-901.E-mail: fabia.alexandra@terra.com.br

\section{Resumo}

Não há consenso na literatura sobre o comportamento clínico-epidemiológico de pessoas com aids de diferentes faixas etárias. Alguns autores defendem que as pessoas com mais de 50 anos de idade são afetadas pela epidemia da aids de forma semelhante às pessoas jovens. O presente estudo propõe-se a comparar características sociodemográficas e clínico-epidemiológicas dos casos de aids com idade igual ou maior que 50 anos, com aqueles de 20 a 39 anos, residentes em Pernambuco e diagnosticados entre $1^{\circ}$ de janeiro de 1990 e 31 de dezembro de 2000. Desenvolveu-se um estudo observacional, do tipo seccional, de base populacional, com dados secundários dos casos notificados à Secretaria de Saúde do Estado de Pernambuco, seguindo-se de revisão de prontuários médicos. Foram incluídos 3.565 casos de aids, sendo $10,8 \%$ com 50 anos ou mais e $89,2 \%$ com 20 a 39 anos. As taxas de incidências ano a ano comportaram-se de maneira semelhante para os dois grupos etários, ao longo do período estudado, exceto para os dois últimos anos, com um incremento do grupo com mais de 50 anos. Estimou-se as razões de prevalência (RP) para as variáveis incluídas, não tendo sido encontradas diferenças em relação ao sexo, contagem de linfócitos $\mathrm{T}$ CD4+ e doenças associadas. Por outro lado, observou-se diferenças estatisticamente significantes entre os grupos para o local de residência $\left(\mathrm{RP}=1,23, \chi^{2}=4,45, \mathrm{p}=0,03\right)$, escolaridade $\left(\mathrm{RP}=1,48, \chi^{2}=4,49, \mathrm{p}=0,03\right)$, categoria de exposição ao $\mathrm{HIV}\left(\mathrm{RP}=1,81, \chi^{2}=\right.$ $27,77, \mathrm{p}<0,001)$ e status vital $\left(\mathrm{RP}=1,32, \chi^{2}=\right.$ $7,69, \mathrm{p}<0,001)$. Os resultados apontam para um aumento gradativo, embora lento, da epidemia de aids na faixa etária de 50 a 70 anos, com predomínio de mais baixa escolaridade e de prática heterossexual. As questões relacionadas a aids e envelhecimento devem ser aprofundadas, principalmente pelo aumento da expectativa de vida da população brasileira, de modo a reorientar ações de prevenção e controle da infecção pelo HIV.

Palavras-chave: Aids. Envelhecimento. Epidemiologia. Prevenção. 


\section{Abstract}

There is no consensus in the literature on the clinical-epidemiological trend of individuals with AIDS in different age groups. Some authors say that AIDS equally affects individuals who above 50 years of age and younger individuals. This study compares socio-demographic and clinical-epidemiologic characteristics of individuals 50 years old and older with the 20-39-year-old group. The individuals are residents of Pernambuco and were diagnosed with AIDS between January 1st, 1990 and December 31st, 2000. An observational, crosssectional, population-based study was developed with secondary data from cases reported to the health department of the state of Pernambuco and through chart review. The sample comprised 3,565 cases, $10.8 \% 50$ years or more, and $89.2 \%$ between 20-39 years of age. The annual incidence was similar in both groups, except in the last two years when there was a slight decrease in the younger group. Prevalence (RP) was estimated for the variables included. Differences were not found in relation to gender, lymphocyte $\mathrm{T}$ (CD4) count, and co-morbidities. On the other hand, a significant difference was observed between groups in relation to site of residence ( $\mathrm{RP}=1.23, \chi^{2}=4.45, \mathrm{p}=0.03$ ), educational level $\left(\mathrm{RP}=1.48, \chi^{2}=4.49, \mathrm{p}=0.03\right)$, HIV exposure $\left.\mathrm{RP}=1.81, \chi^{2}=27.77, \mathrm{p}<0.001\right)$, and vital status $\left(\mathrm{RP}=1.32, \chi^{2}=7.69, \mathrm{p}<0.001\right)$. Results showed a gradually slow increase in the AIDS epidemic in the 50-70-year-old group, with predominance of a lower educational level and heterosexual choice. Questions related to AIDS and aging need to be deepened, mainly due to the increase in life expectancy of the Brazilian population, in order to reorient HIV prevention and infection control actions.

Keywords: Aids. Aging. Epidemiology. Prevention.

\section{Introdução}

A problemática do envelhecimento e aids no Brasil passa por uma questão cultural e de exclusão e concentra-se principalmente no preconceito social relacionado ao sexo nessa idade. A prevenção às doenças sexualmente transmissíveis (DST) e aids entre os maiores de 50 anos é algo muito complexo e representa um desafio para as atuais políticas de saúde pública, que concentram sua atenção na população jovem (entre 20 e 34 anos) ${ }^{1}$.

Estudos comportamentais revelam que o desejo sexual permanece nas pessoas mais idosas ${ }^{2}$ e que a concepção arraigada na sociedade de que sexo é prerrogativa da juventude, contribui para manter fora das prioridades de prevenção das DST e aids os grupos populacionais com idade superior a 50 anos.

É possível que o aumento da expectativa de vida, com os idosos vivendo mais e melhor ${ }^{3}$, e com a disponibilidade de medicamentos que melhoram o desempenho sexual, principalmente dos homens, as pessoas mais idosas sintam-se mais seguras nas investidas amorosas. Para Caldas \& Gessolo ${ }^{1}$, o problema é que a mensagem do sexo sem limitações não veio acompanhada de educação para o uso do preservativo $^{2}$, o que, de certa maneira, revela a omissão da problemática sobre as pessoas mais velhas na abordagem das campanhas educativas de prevenção da aids.

Enquanto a aids é freqüentemente percebida como uma doença de pessoas na idade reprodutiva, alguns estudos revelam o aumento gradual no número de casos, em ambos os sexos, em indivíduos acima de 50 anos de idade ${ }^{1,2,4-7}$. Segundo Linsk ${ }^{8}$, pessoas com mais de 50 anos são afetadas pela epidemia da aids de forma semelhante às pessoas jovens.

O número de casos confirmados de aids com idade acima de 50 anos cresce, no Brasil, como em nenhuma outra faixa etária. Entre os homens, a expansão foi de $98 \%$ na última década. Sobre a parcela feminina idosa, a epidemia avança como um 
rolo compressor: houve um crescimento de $567 \%$ entre 1991 e $2001^{9}$. O aumento expressivo no número de pessoas com mais de 50 anos emerge como um grande desafio para o Brasil, exigindo o estabelecimento de políticas públicas e estratégias que possam garantir qualidade de vida a essas pessoas. Questões como a aids no envelhecimento necessitam de um maior aprofundamento no intuito de fornecer subsídios, tanto para os cuidados com os portadores do vírus da imunodeficiência humana (HIV) e da aids, como para o desenvolvimento de ações e programas de prevenção.

O presente estudo analisa as características sociodemográficas e clínicoepidemiológicas dos casos de aids com idade igual ou maior que 50 anos comparados com os de 20 a 39 anos, residentes em Pernambuco, e diagnosticados entre $1^{\circ}$ de janeiro de 1990 e 31 de dezembro de 2000.

\section{Material e Métodos}

Trata-se de um estudo observacional descritivo, do tipo seccional, de base populacional, utilizando-se dados secundários dos casos de aids notificados à Secretaria de Saúde de Pernambuco.

O estudo foi realizado no estado de Pernambuco, segundo maior estado da Região Nordeste do Brasil, com 185 municípios. Para fins desse estudo, os municípios foram agregados em três regiões: Recife; Região Metropolitana do Recife - RMR (exceto Recife), e Interior.

Foram incluídos todos os casos de aids que se enquadravam nos critérios de definição adotados pelo Ministério da Saúde do Brasil, em vigor na época do estudo ${ }^{10}$, de residentes em Pernambuco, com data de diagnóstico de $1^{\circ}$ de janeiro de 1990 a 31 de dezembro de 2000, notificados à Secretaria de Saúde de Pernambuco (SES/PE) e registrados no Sistema de Informações sobre Agravos de Notificações (SINAN).

Do total de 4.707 casos registrados no SINAN, no período elegível do estudo, fo- ram excluídos 1.142 casos que não atendiam aos critérios de inclusão.

O estudo teve como variável dependente os casos de aids categorizados em dois grupos: 50 anos e mais (grupo caso) e de 20 a 39 anos (grupo de comparação). Como variáveis independentes: escolaridade (analfabeto, $1^{\circ}$ grau - ensino fundamental, $2^{\circ}$ grau - ensino médio e superior); sexo (masculino e feminino); razão de sexo (M:F); local de residência (Recife, RMR e Interior); categoria de exposição (Homossexual masculino - HMS, Bissexual masculino - BSX, Heterossexual - HTS, Usuário de droga injetável - UDI, Hemofílico e Transfusão de sangue); doenças associadas (sarkoma de Kaposi, tuberculose, candidíase, herpes, criptococose, pneumonia por pneumocistis carinni, neurotoxoplasmose, protozoários. Foi também realizado o agrupamento de algumas delas, tomando-se como referência o estudo de Guimarães ${ }^{11}$, acrescentando-se a citomegalovirose); contagem de linfócitos TCD4+ (contagem $<200 \mathrm{cél} / \mathrm{mm}^{3}$ de sangue, contagem $\geq$ a $200 \mathrm{cél} / \mathrm{mm}^{3} \mathrm{e}<350 \mathrm{cél} /$ $\mathrm{mm}^{3}$ de sangue, contagem $\geq 350 \mathrm{cél} / \mathrm{mm}^{3}$ de sangue); status vital (vivo ou morto, até a data da coleta dos casos).

Para melhor demonstrar o efeito das variáveis explicativas na análise de prevalência foram criados estratos (Quadro 1), sendo o nível 1 para cada uma das variáveis, o nível de exposição.

Procedeu-se a revisão de prontuários e registros médicos para complementar os dados das variáveis selecionadas, que se encontravam sem preenchimento na ficha de investigação do SINAN, em cerca de $40 \%$ dos casos, para as variáveis escolaridade, categoria de exposição, doenças/infecções oportunistas e contagem de linfócitos TCD4+. Não foi realizada a revisão dos prontuários de todos os casos. Por questões operacionais, optou-se por fazêla nos hospitais que concentravam cerca de $80 \%$ das notificações de casos de aids no estado de Pernambuco, quais sejam: Hospital Correia Picanço (com um total de 2532 casos, 951 necessitando de revisão), 
Hospital das Clínicas da Universidade Federal de Pernambuco-UFPE (com um total de 496 casos, 245 necessitando de revisão) e Hospital Universitário Oswaldo Cruz da Universidade de Pernambuco-UPE (com um total de 537 casos, 142 necessitando de revisão). Totalizando 1.338 casos, nesses hospitais, dos quais se conseguiu revisar 1.077 prontuários médicos, o equivalente a $80,5 \%$ dos casos. Os casos de outras unidades notificadoras do Estado foram considerados neste estudo, mas as variáveis que não estavam completas foram consideradas como "sem preenchimento".

Para a atualização da variável óbito, procedeu-se a revisão de todos os casos, a partir de 1994, no Sistema de Informação de Mortalidade (SIM). Para os demais anos considerou-se apenas a informação da notificação contida no banco de dados da Secretaria de Saúde.

A distribuição de freqüências das variáveis foi realizada para os grupos etários estudados e as diferenças observadas foram testadas por meio do teste do qui-quadrado, a um nível de significância de 95\% (erro alfa $=5 \%$ ), utilizando-se o programa Epi-Info (versão 6.04).

Para os 110 casos que apresentaram categorias múltiplas de exposição ao HIV, procedeu-se à reclassificação para categoria única, segundo as normas de hierarquização adotadas para o Brasil e publicadas pelo Programa Nacional de DST e Aids (PN DST e Aids) ${ }^{12}$

Para o cálculo de incidência anual dos casos de aids nos dois grupos etários, no período de 1990 a 2000, foram usadas como denominadores as populações dos censos demográficos de 1980, 1991 e 2000, fornecidos pelo $\mathrm{IBGE}^{13}$, e, nos anos intercensitários, as populações estimadas para o Estado. As estimativas foram feitas por meio do programa PROJETOR (versão 01 - Técnicas Globais), desenvolvido em fevereiro de 1993, pelo Departamento de Epidemiologia da Faculdade de Saúde Pública da Universidade de São Paulo.
A razão de sexo (M:F) foi calculada utilizando-se no numerador os casos de aids do sexo masculino e como denominador os casos femininos.

A taxa de incremento anual acumulada foi calculada tomando-se como base o ano de 1990.

As curvas de tendências por sexo e grupo etário foram estimadas verificando-se o modelo de regressão mais adequado, em nível de 95\% de confiabilidade, utilizandose o programa SPSS versão 8.0.

Razões de prevalências para as variáveis sexo, local de residência, grau de escolaridade, categoria de exposição, contagem de linfócitos $\mathrm{TCD}^{+}$, cada uma das nove doenças mais freqüentes associadas e status vital, foram calculadas utilizandose as categorias listadas no Quadro 1, considerando os casos de 50 anos e mais como grupo caso e os de 20 a 39 anos como o grupo de comparação. Para esse procedimento utilizou-se o programa Epi-Info.

Considerando o tipo de desenho de estudo e, sobretudo, a utilização de um banco de dados secundários, as variáveis selecionadas foram assumidas como sendo consistentes e validadas.

As informações referentes à doença e/ ou infecções associadas à aids, no momento da notificação do caso, foram tomadas como os eventos definidores da doença.

O presente estudo foi submetido e aprovado no Comitê de Ética em Pesquisa do Centro de Ciências da Saúde da UFPE (Parecer no 149/2001 - CEP/CCS/UFPE).

\section{Resultados}

Dos 3.565 casos de aids estudados, $10,8 \%$ correspondiam a pessoas com idade igual ou superior a 50 anos, e $89,2 \%$ a pessoas com idade entre 20 e 39 anos.

A análise dos casos segundo os dois grupos etários não mostrou diferenças de distribuição por sexo. Tanto entre o grupo etário mais jovem (20 a 39 anos) quanto entre o mais velho (50 anos e mais), mais de $75 \%$ dos casos são do sexo masculino $\left(\chi^{2}=0,49 p=0,428\right)$. 
Quadro 1 - Categorização das variáveis independentes para o cálculo das razões de prevalência.

Box 1 - Classification of independent variables for the calculation of the prevailing ratio.

\begin{tabular}{|lll|}
\hline Nome da Variável & Nível & Significado \\
\hline Sexo & 1 & Masculino \\
Local de Residência & 2 & Feminino \\
& 1 & Recife \\
Grau de Escolaridade & 2 & RMR + Interior (Demais municípios) \\
& 1 & Analfabeto \\
Categoria de exposição & 1 & $1^{\circ}$ grau $+2^{\circ}$ grau + superior (Demais) \\
& 2 & Heterossexual (HTS) \\
Contagem CD4+ & 1 & Homens que fazem sexo com homens (HSH) \\
& 2 & Menor que 200 cél./mm $\mathrm{mm}^{3}$ \\
Candidíase* & 1 & Igual ou superior a 200 cél./mm $\mathrm{mm}^{3}$ \\
& 2 & Não \\
Status vital & 1 & Morto \\
& 2 & Vivo \\
\hline
\end{tabular}

*Procedeu-se a mesma estratificação para as demais doenças

A distribuição dos casos ano a ano, no período de 1990 a 2000 comportou-se de maneira semelhante quando comparados os dois grupos etários. No geral observase um crescimento anual de casos nas duas faixas etárias consideradas no estudo. Vale ressaltar que o número de casos de aids no grupo mais velho (50 anos e mais), em Pernambuco, aumentou de 19 casos em 1990 para 57 casos em 2000, o que corresponde ao aumento da taxa de incidência por 100.000 habitantes de 4,2 para 9,5 e um incremento acumulado de $200 \%$. Esse incremento foi mais expressivo especialmente a partir do ano de 1996. Foi observado, ainda, que o incremento de casos entre os jovens (20 a 39 anos) apresentou diminuição nos últimos anos, ao contrário do grupo mais velho (50 anos e mais), conforme mostra a Tabela 1.

A análise das curvas de incidências no grupo mais velho (50 anos e mais) revelou que, no período entre 1990 e 1995, as incidências foram estáveis em ambos os sexos, tendo um crescimento significativo após 1995, chegando a uma incidência de 7,1 casos por 100.000 habitantes em 2000, no sexo masculino. Em relação ao sexo feminino, a incidência em 1990, que era de 0,2 casos em 100.000 habitantes, passou em 2000 para 2,4 casos.

Um comportamento exponencial ( $\mathrm{p}<$ $0,005)$ das incidências no sexo masculino e no total de Pernambuco foi revelado por ocasião da análise das curvas de regressão, diferentemente para o sexo feminino, onde não foi possível demonstrar este tipo de distribuição. Isso se deve, provavelmente, ao fato de ter sido constatado um grande número de incidências muito próximas de zero no sexo feminino, inviabilizando o modelo. Neste sentido, foram testados outros modelos de curvas (linear, S e cúbica), mas em nenhumas delas obteve-se o resultado esperado (Gráfico 1).

Ao proceder à mesma análise no grupo etário mais jovem (20 a 39 anos), obteve-se um crescimento na incidência até 1998 para ambos os sexos e, conseqüentemente, na incidência total, com uma discreta redução nos anos de 1999 e 2000. As curvas de regressão para esse grupo etário mais jovem apresentaram um comportamento exponencial $(p<0,005)$, conforme demonstrado no Gráfico 2.

Recife apresentou 53,9\% de casos de aids em pessoas com 50 anos e mais, e o 
Tabela 1 - Número absoluto de casos de aids, percentual de incremento e incidência anual por 100.000 habitantes, segundo grupo etário. Pernambuco, 1990 a 2000.

Table 1 - Absolute number of AIDS cases, percentage of increment and annual incidence per 100.000 inhabitants, according age group. Pernambuco, 1990 to 2000.

\begin{tabular}{|c|c|c|c|c|c|c|c|}
\hline \multirow{3}{*}{$\begin{array}{l}\text { Ano de } \\
\text { Diagnóstico }\end{array}$} & \multicolumn{5}{|c|}{ Grupo etário } & \multicolumn{2}{|c|}{ Total } \\
\hline & \multicolumn{2}{|c|}{50 anos e mais } & \multicolumn{3}{|c|}{20 a 39 anos } & & \\
\hline & $\Delta^{*}(\%)$ & Inc. & $\mathrm{N}$ & $\Delta^{*}(\%)$ & Inc. & & \\
\hline 1990 & 19 & - & 4,2 & 118 & - & 12,2 & 137 \\
\hline 1991 & 14 & $-26,3$ & 3,1 & 167 & $+41,5$ & 16,7 & 181 \\
\hline 1992 & 18 & $-5,3$ & 3,8 & 187 & $+58,5$ & 18,3 & 205 \\
\hline 1993 & 21 & $+10,5$ & 4,3 & 224 & $+89,8$ & 21,5 & 245 \\
\hline 1994 & 20 & $+5,3$ & 4,1 & 259 & $+110,5$ & 24,2 & 279 \\
\hline 1995 & 29 & $+52,6$ & 5,5 & 267 & $+126,3$ & 24,5 & 296 \\
\hline 1996 & 58 & $+205,3$ & 10,8 & 365 & $+209,3$ & 32,5 & 423 \\
\hline 1997 & 43 & $+126,3$ & 7,9 & 395 & $+234,7$ & 34,2 & 438 \\
\hline 1998 & 52 & $+173,7$ & 9,3 & 476 & $+303,4$ & 40,1 & 528 \\
\hline 1999 & 53 & $+178,9$ & 9,0 & 377 & $+219,5$ & 31,1 & 430 \\
\hline 2000 & 57 & $+200,0$ & 9,5 & 346 & $+193,2$ & 27,9 & 403 \\
\hline Total & 384 & - & & 3181 & - & & 3565 \\
\hline
\end{tabular}

*Incremento acumulado; tomou-se como base de cálculo o ano de 1990

*Accumulated increase; year of 1990 was used for calculation

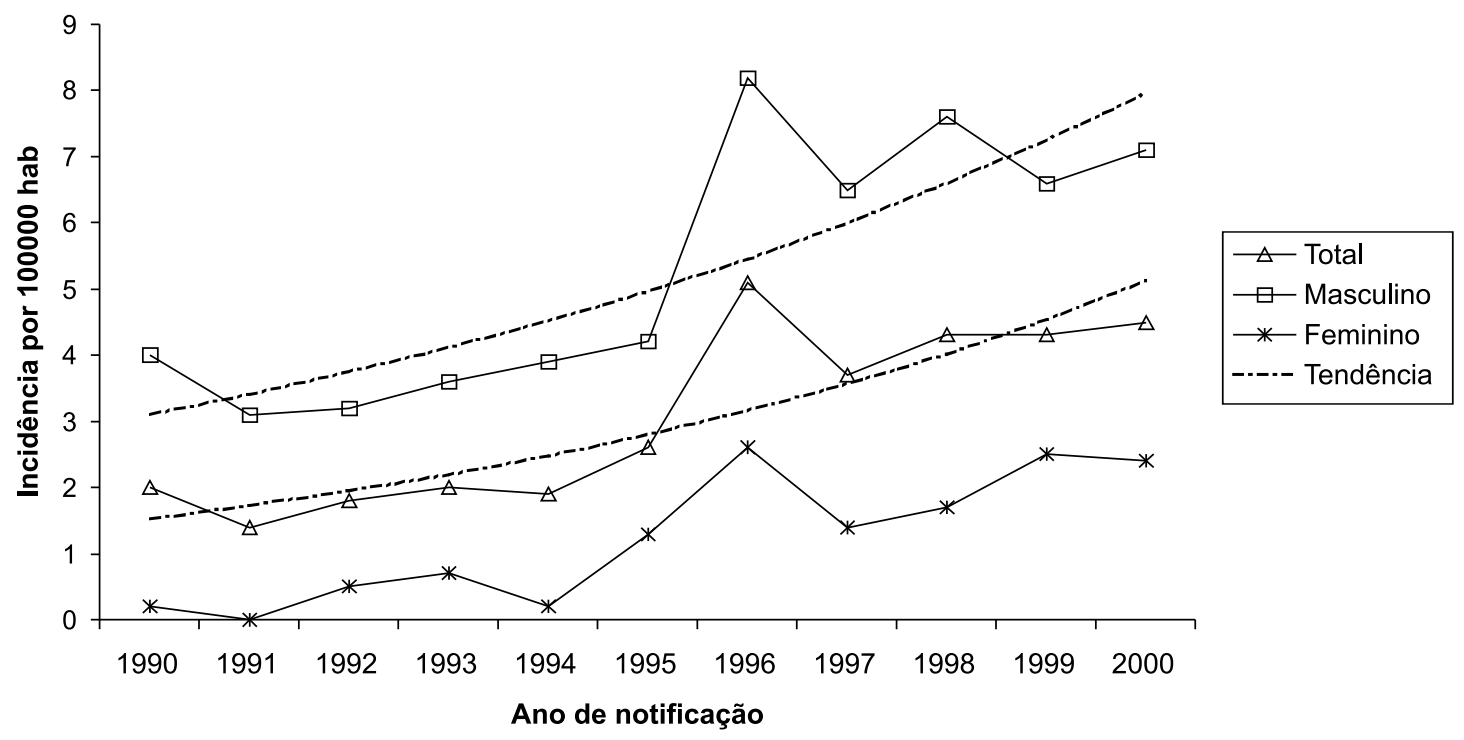

Gráfico 1 - Incidência de casos de aids no grupo etário de 50 anos e mais por ano de diagnóstico e sexo. Pernambuco, 1990 a 2000.

Chart 1 - Incidence of cases of AIDS in the group of 50 years and more of diagnosis and sex. Pernambuco, 1990 to 2000.

grupo etário mais jovem respondeu por 48,1\% dos casos do município; já para os residentes da RMR (excluindo-se o município do Recife), foi verificada a predominância entre os mais jovens (27,6 versus 33,6) (Tabela 2).
Independentemente da faixa etária, a maioria absoluta dos casos ocorreu por transmissão sexual, 96,6\%; apenas 3,4\% resultaram da transmissão sanguínea. $\mathrm{Na}$ categoria de transmissão sexual, observouse predomínio da subcategoria heterosse- 


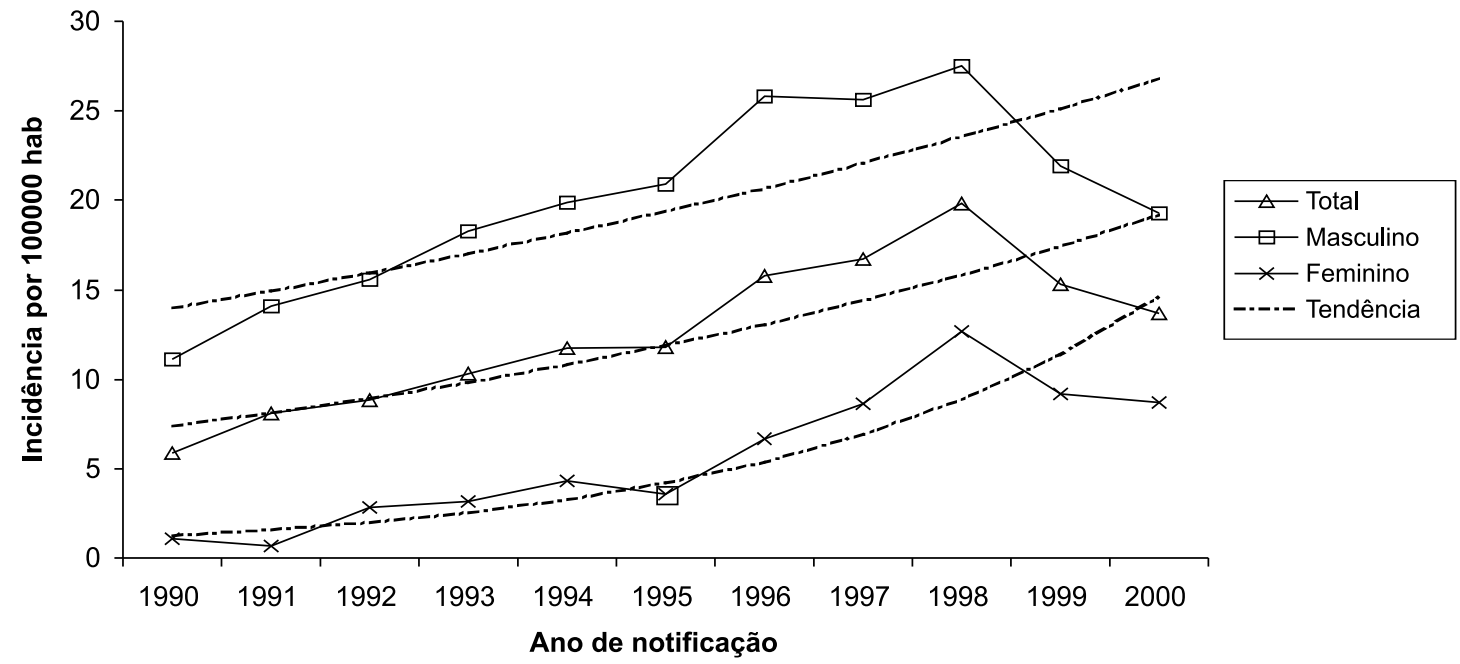

Gráfico 2 - Incidência de casos de aids no grupo de 20 a 39 anos por ano de diagnóstico e sexo. Pernambuco, 1990 a 2000.

Chart 2 - Incidence of cases of AIDS in the group of 20 a 39 years per year of diagnosis and sex. Pernambuco, 1990 to 2000.

Tabela 2 - Número absoluto e relativo (\%) dos casos de aids segundo local de residência e grupo etário. Pernambuco, 1990 a 2000.

Table 2 - Absolute and relative number (\%) of the cases according local AIDS as of residence and age group. Pernambuco, 1990 to 2000.

\begin{tabular}{lcccccc}
\hline & \multicolumn{4}{c}{ Grupo etário } & \multicolumn{3}{c}{ Total } \\
Local de & 50 anos e mais & \multicolumn{2}{c}{20 a 39 anos } & $\mathrm{n}$ & $\%$ \\
residência & $\mathrm{n}$ & $\%$ & $\mathrm{~N}$ & $\%$ & 1736 & 48,7 \\
\hline Recife & 207 & 53,9 & 1529 & 48,1 & 1736 \\
RMR & 106 & 27,6 & 1068 & 33,6 & 1174 & 32,9 \\
Interior & 71 & 18,5 & 584 & 18,4 & 655 & 18,4 \\
\hline Total & 384 & 100 & 3181 & 100 & 3565 & 100 \\
\hline
\end{tabular}

$\chi^{2}=6,11 \mathrm{p}=0,047$

xual na faixa etária de 50 anos e mais (57,6\%) (Tabela 3).

A análise dos casos que apresentavam a variável escolaridade preenchida nas duas faixas etárias do estudo, revelou que a maioria dos indivíduos tinha baixa escolaridade (ou seja, cursou no máximo o ensino fundamental ou $1^{\circ} \mathrm{Grau}$ ), sendo $57,5 \%$ no grupo mais velho e $59,1 \%$ nos mais jovens (Gráfico 3).

A análise do status vital revelou um maior percentual de óbitos no grupo etário com idade igual ou superior a 50 anos (60\%). A diferença encontrada foi estatisticamente significante (Tabela 4).

\section{Estudo de prevalência}

Para o cálculo das razões de prevalência entre o grupo etário mais velho (50 anos e mais) e o grupo etário mais jovem (20 a 39 anos) e cada uma das variáveis explicativas incluídas no estudo, foram criadas variáveis categóricas conforme demonstrado anteriormente no Quadro 1. Os resultados obtidos estão apresentados na Tabela 5 .

A análise individual de cada variável, segundo a distribuição de casos de aids nos dois grupos etários (Tabela 5), revelou que as diferenças obtidas para sexo, contagem de linfócitos $\mathrm{TCD}^{+}$e cada uma das nove 
Tabela 3 - Número absoluto e relativo (\%) dos casos de aids segundo categoria de exposição e grupo etário. Pernambuco, 1990 a 2000.

Table 3 - Absolute and relative number (\%) of the cases of AIDS according category of exposition and age group. Pernambuco, 1990 to 2000.

\begin{tabular}{lcccccc}
\hline & \multicolumn{3}{c}{ Grupo etário } & & \multicolumn{2}{c}{ Total $^{*}$} \\
Categoria de & 50 anos e mais & \multicolumn{2}{c}{20 a 39 anos } & N & $\%$ \\
Exposição & $\mathrm{N}$ & $\%$ & $\mathrm{~N}$ & $\%$ & 2826 & 96,6 \\
\hline Sexual & 294 & 97,3 & 2532 & 96,5 & 1044 & 36,9 \\
\hline H M S & 67 & 22,2 & 977 & 37,2 & 518 & 18,3 \\
B S X & 53 & 17,5 & 465 & 17,7 & 1264 & 44,2 \\
H T S & 174 & 57,6 & 1090 & 41,6 & 99 & 3,4 \\
\hline Sanguínea & 8 & 2,7 & 91 & 3,5 & 70 & 2,4 \\
\hline U D I & - & - & 70 & 2,7 & 10 & 0,3 \\
Hemofílico & 1 & 0,3 & 9 & 0,3 & 19 & 0,7 \\
Hemotransfusão & 7 & 2,4 & 12 & 0,5 & 2925 & 100,0 \\
\hline Total & 302 & 100,0 & 2623 & 100,0 & \\
\hline
\end{tabular}

*Em 640 (18\%) casos não havia registro da informação" forma de exposição ao HIV" $\chi^{2}=55,87 \mathrm{p}=0,000$

* $640(18 \%)$ cases did not have a record of the route of exposure to HIV $c 2=55.87 \mathrm{p}=0.000$

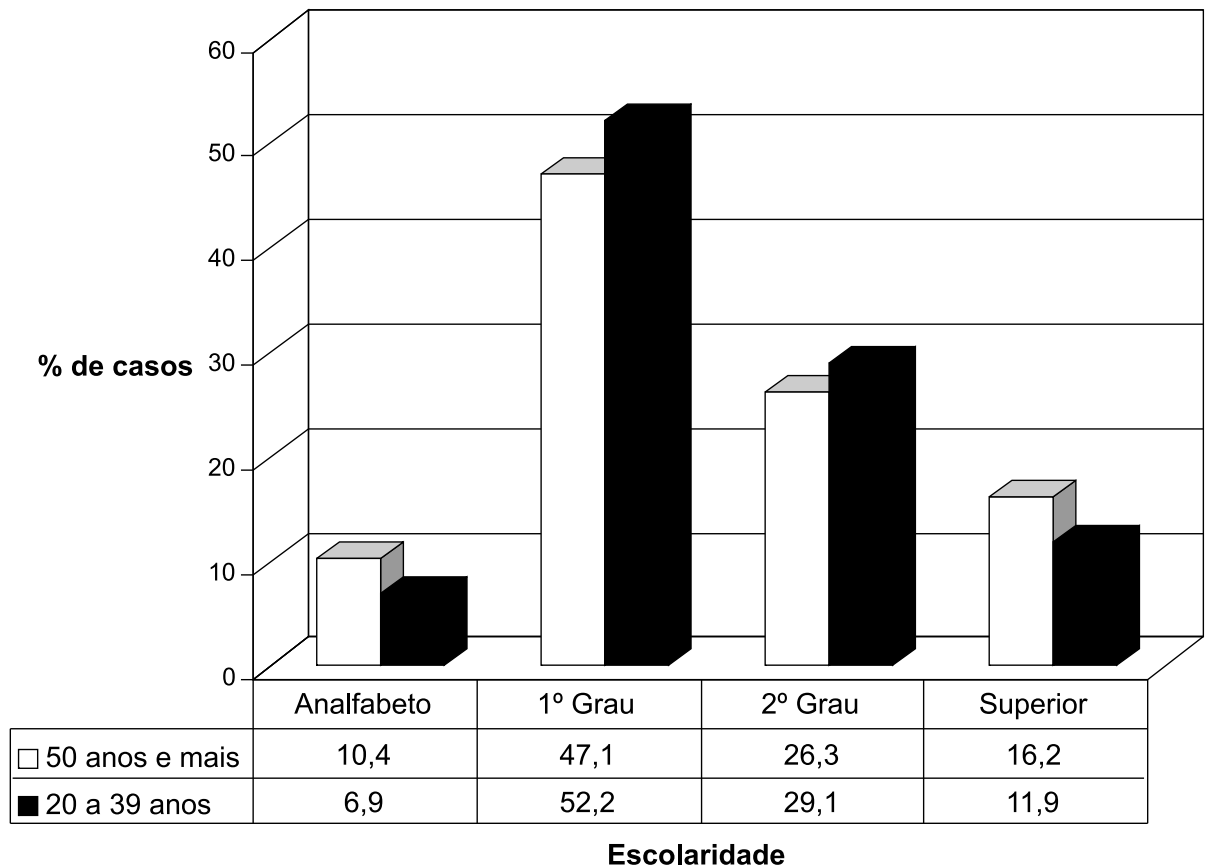

*Em 703 (19,7\%) casos não havia registro da informação da escolaridade $\chi^{2}=11,0 p=0,0117$

*Information on schooling could not be obtained for 703 (19.7\%) cases

Gráfico 3 - Percentual dos casos de aids segundo grupo etário e nível de escolaridade. Pernambuco, 1990 a 2000.

Chart 3 - Percentage of AIDS cases according to age group and level of schooling. Pernambuco, 1990 to 2000. 
Tabela 4 - Número absoluto e relativo (\%) dos casos de aids segundo status vital e grupo etário. Pernambuco, 1990 a 2000.

Table 4 - Absolute and relative number (\%) of the cases of AIDS according vital status and age group. Pernambuco, 1990 to 2000.

\begin{tabular}{lcccccc}
\hline & \multicolumn{4}{c}{ Grupo etário } & \multicolumn{3}{c}{ Total } \\
Status & 50 anos e mais & \multicolumn{2}{c}{20 a 39 anos } & \multicolumn{2}{c}{} \\
Vital* & $\mathrm{n}$ & $\%$ & $\mathrm{n}$ & $\%$ & 1652 & 46,3 \\
\hline Vivo & 152 & 40,0 & 1500 & 47,7 & 1875 & 52,6 \\
Morto & 228 & 60,0 & 1647 & 52,3 & 3527 & 100,0 \\
\hline Total & 380 & 100,0 & 3147 & 100,0 & 3 \\
\hline
\end{tabular}

* Em $38(1,1 \%)$ casos não havia registro da informação do status vital.

$\chi^{2}=7,69 p=0,005$

* $38(1.1 \%)$ cases did not have information on the vital status.

$\chi^{2}=7.69 p=0.005$

doenças associadas não foram estatisticamente significantes.

\section{Discussão}

O valor encontrado neste estudo $(10,8 \%)$ para os casos de aids na faixa etária de 50 anos e mais assemelha-se a outros estudos desenvolvidos em diferentes locais, com cortes de idade semelhantes $^{5,6,8,14-16}$

No Brasil, a notificação dos casos de aids passou a ser obrigatória a partir de 1986, e ainda assim o sistema de Vigilância Epidemiológica da aids padece com a subnotificação e com o atraso nas notificações (sendo esta a maior falha apontada no sistema). Esta é uma das justificativas plausíveis para a discrepância entre o tamanho da epidemia obtida com os casos notificados e a verdadeira magnitude da mesma. Sabe-se que a subnotificação e o atraso de notificação podem variar entre as diferentes regiões e períodos de tempo $^{12,17,18}$

A qualidade da informação é um dos fatores que limitam o uso de dados secundários para o entendimento da situação de saúde da população. Mesmo após a revisão de outras fontes de dados, em busca de complementação de informações não preenchidas na ficha de notificação de casos, houve a permanência de um grande número de informações ignoradas, o que limitou e comprometeu a análise de algu- mas características propostas no estudo.

Neste estudo, a discreta redução encontrada no grupo etário mais jovem, em ambos os sexos, nos anos de 1999 e 2000, deve-se, provavelmente, ao atraso da notificação.

Atualmente, a faixa etária de 50 a 59 anos responde pela segunda maior incidência de aids, por idade, no país. Desde 1994, este grupo etário sofreu um incremento da taxa de incidência, chegando a ultrapassar a faixa etária de 30 a 39 anos, que apresentou um crescimento do número de casos em menor velocidade. Uma outra importante mudança percebida foi que, a partir de 1995, houve um crescimento da incidência na faixa etária de 60 a 69 anos (16,22 casos/100.000 habitantes), que passou a ser a quarta faixa etária de maior incidência de casos no país ${ }^{4}$

A tendência de feminização da epidemia da aids é um tema que há alguns anos vem sendo debatido em todo o país e em outras partes do mundo ${ }^{19,20}$. Neste estudo, embora o número de casos do sexo masculino tenha sido maioria nas duas faixas etárias, foi observada uma notória expansão da epidemia entre as mulheres. Essa mesma tendência do crescimento de casos femininos foi observada na faixa etária com idade igual ou superior a 50 anos. Em Pernambuco, a razão de sexo, que era 19:1 no ano de 1994, declinou progressivamente para 2,1 e 2,4 casos masculinos para cada caso feminino, em 1999 e 2000, respecti- 
Tabela 5 - Razão de prevalência (RP) dos casos de aids. Pernambuco, 1990 a 2000.

Table 5 - Reason of prevalence (RP) of the cases of aids. Pernambuco, 1990 to 2000.

\begin{tabular}{|c|c|c|c|c|c|}
\hline Nome da Variável & Estrato de risco & $\mathrm{RP}$ & IC & $\chi^{2}$ & Valor $p$ \\
\hline Sexo & $\begin{array}{l}\text { Masculino } \\
\text { Feminino }\end{array}$ & 1,09 & $(0,87 ; 1,37)$ & 0,49 & 0,48 \\
\hline Local de Residência & $\begin{array}{l}\text { Recife } \\
\text { Demais }\end{array}$ & 1,23 & $(1,02 ; 1,49)$ & 4,45 & 0,03 \\
\hline Grau de Escolaridade & $\begin{array}{l}\text { Analfabeto } \\
\text { Demais }\end{array}$ & 1,48 & $(1,06 ; 2,07)$ & 4,49 & 0,03 \\
\hline Categoria de exposição & $\begin{array}{l}\mathrm{HTS} \\
\mathrm{HSH}\end{array}$ & 1,81 & $(1,45 ; 2,25)$ & 27,77 & 0,00 \\
\hline Contagem CD4+ & $\begin{array}{l}<200 \text { cél. } / \mathrm{mm}^{3} \\
\geq 200 \text { cél. } / \mathrm{mm}^{3}\end{array}$ & 1,03 & $(0,75 ; 1,43)$ & 0,01 & 0,91 \\
\hline Candidíase & $\begin{array}{l}\text { Sim } \\
\text { Não }\end{array}$ & 0,92 & $(0,75 ; 1,13)$ & 0,53 & 0,46 \\
\hline Neurotoxoplasmose & $\begin{array}{l}\text { Sim } \\
\text { Não }\end{array}$ & 0,87 & $(0,68 ; 1,12)$ & 1,03 & 0,31 \\
\hline PPC & $\begin{array}{l}\text { Sim } \\
\text { Não }\end{array}$ & 0,94 & $(0,75 ; 1,17)$ & 0,25 & 0,62 \\
\hline CMV & $\begin{array}{l}\text { Sim } \\
\text { Não }\end{array}$ & 0,73 & $(0,37 ; 1,43)$ & 0,60 & 0,43 \\
\hline Herpes simples & $\begin{array}{l}\text { Sim } \\
\text { Não }\end{array}$ & 1,25 & $(0,86 ; 1,81)$ & 1,08 & 0,29 \\
\hline Cripotococose & $\begin{array}{l}\text { Sim } \\
\text { Não }\end{array}$ & 0,91 & $(0,42 ; 1,96)$ & 0,00 & 0,97 \\
\hline Protozooses & $\begin{array}{l}\text { Sim } \\
\text { Não }\end{array}$ & 1,08 & $(0,71 ; 1,63)$ & 0,05 & 0,82 \\
\hline Sarcoma Kaposi & $\begin{array}{l}\text { Sim } \\
\text { Não }\end{array}$ & 1,24 & $(0,78 ; 1,97)$ & 0,54 & 0,46 \\
\hline Tuberculose & $\begin{array}{l}\text { Sim } \\
\text { Não }\end{array}$ & 0,78 & $(0,54 ; 1,15)$ & 1,37 & 0,24 \\
\hline Status vital & $\begin{array}{l}\text { Morto } \\
\text { Vivo }\end{array}$ & 1,32 & $(1,09 ; 1,61)$ & 7,69 & 0,00 \\
\hline
\end{tabular}

vamente. Estudos semelhantes como o de Santana e colaboradores ${ }^{21}$, Sanchez ${ }^{22}$, Matsushita e Santana ${ }^{4}$, corroboraram os resultados encontrados neste trabalho. Todos eles comprovaram a feminização da epidemia nos últimos anos.

O predomínio encontrado na proporção de casos de aids em residentes no Recife, no grupo caso (50 anos e mais) em relação ao grupo etário mais jovem, e a verificação do oposto nas outras regiões, aponta para a possibilidade de estabilização da aids no Recife, epicentro da epidemia em Pernambuco. A predominância dos casos em pessoas mais jovens nos municípios pequenos do Estado, especialmente aqueles com menos de 50.000 habitantes, pode indicar fase inicial de expansão da epidemia nessas localidades.

Mesmo na ocorrência de importantes transformações no perfil da aids no Brasil, como, por exemplo, a interiorização que 
ocorre sobretudo por conta da difusão geográfica da doença a partir dos grandes centros urbanos em direção aos municípios de médio e pequeno porte do interior do país, pode-se perceber, claramente, que existem dinâmicas regionais e populacionais distintas ${ }^{20}$. Em pesquisa realizada no Estado do Rio de Janeiro com os casos de aids em pessoas com mais de 60 anos, entre 1995 e 2001, observou-se que $68,2 \%$ dos casos eram de residentes da capital do Estado ${ }^{22}$.

Acompanhando a progressão da epidemia da aids no Brasil e em outras regiões do mundo, observa-se, cada vez mais, um aumento de casos em decorrência da transmissão sexual, embora também se registre uma tendência de crescimento entre $\mathrm{UDI}^{20,33,24}$. Segundo Prilip ${ }^{9}$, até meados da década de 80 , a falta de métodos para seleção de doadores e controle de sangue faziam com que a transmissão sangüínea representasse o principal fator de risco para a transmissão do HIV em pessoas com mais de 60 anos. Esta mesma autora coloca ainda que, atualmente, a maioria dos casos de aids em pacientes desta faixa etária é atribuída ao contato sexual ou ao uso de drogas injetáveis.

A epidemia da aids em Pernambuco tem como característica predominante a transmissão sexual, que a torna diferente de outras regiões do Brasil, particularmente Sudeste, Centro-Oeste e Sul ${ }^{23}$.

Importantes mudanças ocorreram no país desde o início da epidemia até o momento atual, no que diz respeito à transmissão sexual. À extensa disseminação inicial no segmento de homens que fazem sexo com outros homens (HSH), ou seja, homossexuais e bissexuais masculinos, seguiu-se uma certa estabilização em anos posteriores ao início da epidemia. No ano de $1983,69,2 \%$ dos casos diziam respeito a essas duas categorias de exposição, enquanto em 2001 representou apenas $23,4 \%$ do total de casos notificados desde o início da epidemia ${ }^{12,25}$, assim como o aumento da transmissão HTS, caracterizando a heterossexualização da epidemia ${ }^{23}$.
A transmissão heterossexual constitui, na atualidade, a mais importante característica da dinâmica da epidemia, com expressão relevante em todas as regiões. Essa forma de transmissão vem, ao longo dos anos, apresentando um importante aumento no Brasil, passando de $6,6 \%$ de casos em 1988 para 32,3\% em 2001 $1^{12,20}$.

A região Nordeste é caracterizada pelo predomínio da transmissão heterossexual para ambos os sexos, com baixas proporções de casos registrados em UDI, com aproximadamente $2 \%$ dos casos até 2001 , diferentemente do perfil epidemiológico apresentado em outras regiões do país, sobretudo nas regiões Sul e Sudeste, onde a transmissão pelo uso de drogas injetáveis mantém-se em patamares altos e constantes nos últimos anos ${ }^{26,19}$. Em Pernambuco, no período de 1983-1986, predominava a transmissão do HIV entre HSH com 89,7\% dos casos. No período de 1987-1991, essa mesma sub-categoria passou a represen$\operatorname{tar} 62,1 \%$ dos casos, surgindo nesse período os primeiros casos de aids entre HTS, com $15,7 \%$ dos casos. Desde então a transmissão entre HTS tem apresentado tendência crescente, correspondendo, até abril/2002, a $44,4 \%$ do total de casos no Estado $^{27}$

Verificou-se neste estudo uma maior freqüência da sub-categoria heterossexual, tanto no grupo caso (50 anos e mais) quanto no grupo de comparação (20 a 39 anos) Já na subcategoria homossexual, observouse um predomínio no grupo de jovens. Esses achados, até certo ponto, corroboram com os encontrados por Sanches ${ }^{22}$ no estado do Rio de Janeiro, onde também predominou a subcategoria heterossexual em pessoas com mais de 60 anos, com $46,7 \%$ dos casos entre os homens e $95,5 \%$, entre as mulheres. No presente estudo, chamase a atenção para o fato de não ter havido nenhuma transmissão por UDI no grupo de 50 anos e mais, além da baixa freqüência de casos nas subcategorias hemofílico/ hemotransfusão.

O nível educacional expressa diferenças entre pessoas em termos de acesso à 
informação e perspectivas e possibilidades de se beneficiar de novos conhecimentos. É inegável que alguns parâmetros para alcançar uma maior qualidade de prevenção e assistência, tais como o acesso à educação e aos métodos preventivos, estão diretamente ligados à situação socioeconômica da população.

Dentre os indicadores mais importantes para mensurar o nível socioeconômico associado à saúde, cita-se o nível de instrução, a renda e a ocupação. Essas variáveis têm em comum o fato de evidenciarem a estratificação social, pois os indivíduos ocupam uma posição de hierarquia social de acordo com sua ocupação, renda e nível educacional ${ }^{28,29}$. Destaca-se que, mesmo com restrições, o grau de instrução tem sido utilizado como uma variável auxiliar na tentativa de expressar o perfil socioeconômico dos casos de aids ${ }^{20}$.

O fenômeno da pauperização tem sido caracterizado pelo aumento da proporção de casos de aids em indivíduos de baixa escolaridade. Estudos que discutem a pauperização da epidemia de aids no Brasil revelam que, nos últimos anos, os casos de aids têm ocorrido com maior freqüência em pessoas com baixo nível de escolaridade (analfabetas e apenas $1^{\circ}$ grau $)^{24,28,29}$.

A significativa mudança no perfil de escolaridade dos casos notificados entre adultos e adolescentes é demonstrada com a tendência de aumento de registros de pacientes de menor grau de escolaridade ao longo do tempo. Em 1982, a totalidade dos casos de aids com escolaridade conhecida apresentava nível superior ou segundo grau; em 1985, o percentual deste grupo alcançou $76 \%$, enquanto apenas $24 \%$ dos casos eram analfabetos ou tinham cursado até o primeiro grau; a partir de 1999/ 2000, houve inversão desses valores, sendo que, dos casos com escolaridade informada, $74 \%$ eram analfabetos ou haviam cursado até o primeiro grau, e apenas $26 \%$ tinham cursado até o segundo grau ou tinham escolaridade superior ${ }^{20}$.

As curvas de sobrevida depois do diag- nóstico de aids confirmaram o curso mais rápido da infecção no grupo mais velho, com sobrevida de 1 ano $=35 \%$ versus $86 \%$ para a amostra mais jovem ${ }^{30}$. Estudo conduzido nos Estados Unidos da América ${ }^{7}$ revelou que o tempo de sobrevida dos casos de aids foi de 9 meses no grupo de mais velhos e de 22 meses no grupo de mais jovens. Ship e colaboradores ${ }^{15}$, e Butt e colaboradores $^{31}$ encontraram, também, maior probabilidade dos doentes mais velhos morrerem mais rapidamente quando comparados com doentes mais jovens. Esta maior mortalidade entre os idosos também foi encontrada no presente estudo, onde observou-se diferenças estatisticamente significantes quanto à proporção de óbitos entre o grupo etário mais velho (50 anos e mais) e o grupo etário mais jovem (20 a 39 anos).

Ferro $^{30}$ e Prilip ${ }^{9}$ mostraram que, em pacientes idosos, o diagnóstico é feito em uma fase mais tardia da história natural da infecção pelo HIV. Nos idosos, freqüentemente a infecção pelo HIV só é diagnosticada depois de uma investigação extensa e por exclusão de outras doenças, o que atrasa o diagnóstico e o tratamento. Esse fato pode ser explicado pela ausência de suspeita desta infecção nos pacientes mais velhos, ou também, por eles terem um tempo mais curto entre a infecção e aparecimento da doença devido ao envelhecimento do sistema imunológico.

Não causa surpresa o fato de pessoas idosas não adotarem medidas de prevenção para o HIV, pois, além da questão cultural, os programas de prevenção são mais voltados para a camada da população mais jovem, a exemplo das campanhas veiculadas na mídia.

Na maior parte do mundo, quase todas as mensagens de prevenção são dirigidas a adultos jovens e adolescentes. Na melhor das hipóteses, as ilustrações e mensagens nos materiais de prevenção tendem a ter idade neutra, com imagens envolvendo tipicamente pessoas fisicamente atraentes e mais jovens ${ }^{8}$.

O certo é que a epidemia da aids na fai- 
xa etária de 50 a 70 anos tem demonstrado um aumento gradativo, embora lento, e aparentemente não tem demonstrado certa estabilização como em outras faixas etárias. Essas mudanças poderiam ser explicadas por fatores diversos, ligados à sexualidade, como questões culturais (baixa noção de risco - o próprio idoso se vê como uma pessoa afastada da exposição ao vírus ${ }^{9}$, não realização de sexo seguro), heterossexualização e feminização (ampliando o universo de susceptíveis), terapias mais eficazes (adiamento da instalação e notificação da aids), aumento da expectativa de vida ao nascer e aumento da atividade sexual (reposição hormonal, para as mulheres e tratamento da impotência sexual entre os homens) ${ }^{4}$.

\section{Conclusão}

Os resultados do presente estudo apontam para um aumento gradativo do número de casos de aids entre pessoas com mais de 50 anos, em Pernambuco, com predomínio de pessoas com mais baixa escolaridade e com prática heterossexual.

A adoção de políticas de saúde pública que concentrem sua atenção na população mais velha é necessária para conter o avanço da aids entre os indivíduos com mais de 50 anos. Isso porque o incremento de pessoas mais velhas vitimadas pela aids tende a se ampliar, sobretudo pelo aumento da expectativa de vida e pelo fato dessa faixa da população vir sendo negligenciada, tanto em termos de acesso à informação quanto de um atendimento diferenciado, que leve em consideração aspectos psicológicos, socioeconômicos e culturais que interferem na vulnerabilidade desse grupo etário, fazendo com que a população mais velha fique exposta à contaminação por não ter a percepção do risco.

Também seria de fundamental importância uma articulação entre a Coordenação Nacional de DST/aids e a Área Técnica Saúde do Idoso do Ministério da Saúde, para juntos trabalharem em prol da diminuição dos índices de contaminação pelo HIV e outras doenças sexualmente transmissíveis entre os mais velhos.

A realização de programas de prevenção voltados para o atendimento de pessoas com 50 anos ou mais portadoras de HIV/aids, devem estar atentos às questões de sexualidade no envelhecimento, onde será necessário quebrar tabus: os mais velhos nunca são vistos como pessoas que pensam em sexo. A sexualidade nessa faixa etária não é discutida e, em alguns casos, é até ignorada. Os mais velhos devem ser enxergados como indivíduos que possuem desejos, necessidades sexuais e fazem projetos para o futuro.

\section{Referências}

1. Caldas JMP, Gessolo KM - AIDS depois dos 50: um novo desafio para as políticas de saúde pública; 2006. Disponível em http:/ /www.aidscongress.net/ article.php?id_comunicacao=285 Acessado em 3 de março de 2007.

2. Ribeiro A. Sexualidade na terceira idade. In: Carvalho Filho ET, Papaléo Neto M. Geriatria. São Paulo: Atheneu; 1996. p. 124-34.

3. Lisboa MES - A invisibilidade da população acima de 50 anos no contexto da epidemia de HIV/aids; 2006. Disponível em http://www.aidscongress.net/ article.php?id_comunicacao $=285$ Acessado em 03 de março de 2007.
4. Matsushita RY, Santana RS. Uma análise da incidência dos casos de aids por faixa etária. Boletim Epidemiológico-AIDS. Brasília 2001; 14(2): 3-5.

5. Stall R, Catania J. Aids risk behaviors among late middle aged and elderly americans. Archiv Int Med 1994; 154: 57 63.

6. Martin JN, Colford Jr JM, Ingo L, Tager IB. Effect of older age on survival in human immunodeficiency virus (HIV) disease. Am J Epidemiol 1995; 142: 1221-30.

7. Chen HX, Ryan PA, Ferguson RP, Yataco A, Markowitz JA, Raksis, K. Characteristics of acquired immunodeficiency syndrome in older adults. JAm Geriatr Soc 1998; 46: 153-7. 
8. Linsk NL. HIV among older adults: age-specific issues in prevention and treatment. Aids Reader 2000; 10: 430-40.

9. Prilip NBA. Aids atinge idosos. Disponível em http:// www.portaldoenvelhecimento. net/pforum/aids2.htm. Acessado em 2 de março de 2007.

10. Brasil. Coordenação Nacional de DST/aids. Revisão da definição nacional de caso de aids em indivíduos maiores de 13 anos para fins de vigilância epidemiológica. Brasília; 1998.

11. Guimarães MDC. Estudo temporal das doenças associadas à aids no Brasil. Cad Saúde Pública 2000; 16: 21-36.

12. Brasil. Editorial. Boletim Epidemiológico - AIDS, Brasília, ano 15, n.1; 2001.

13. Instituto Brasileiro de Geografia e Estatística - IBGE. Censo demográfico 2000. Rio de janeiro: IBGE; 2000.

14. Moss RJ, Miles SH. Aids and geriatrician. JAm Geriatr Soc 1987; 35: 460-4.

15. Ship JA, Wolff A, Selik RM. Epidemiology of acquired immune deficiency syndrome in persons aged 50 years or older. J Acquir Immune Defic Syndr 1991; 4: 84-8.

16. Kernutt GJ, Price AJ, Judd FK, Burrows GD. Human immunodeficiency virus infection, dementia and the older patients. Aust NZ J Psychiatry 1993; 27: 9-19.

17. Castilho E, Szwarcwald CL. A Aids: mais uma pedra no caminho dos jovens brasileiros. Rio de Janeiro: FIOCRUZ; 1997.

18. Luiz RR, Costa AJL. Sobre a correção do atraso de notificação dos casos de aids no Brasil. Boletim Epidemiológico - AIDS. Brasília 2001; 14(2).

19. Szwarcwald CL, Bastos FI, Esteves MAP, Andrade, CLT. A disseminação da epidemia da aids no Brasil, no período de 1987-1996: uma análise espacial. Cad Saúde Pública 2000; 16: 7-19.

20. Brito AM, Castilho EA, Szwarcwald CL. Aids e infecção pelo HIV no Brasil: uma epidemia multifacetada. Rev Soc Bras Med Trop 2001; 2: 207-17.
21. Santana R, Dhalia C, Barreira D, Barbosa A. Aids na faixa etária maior que 50 anos. Pontos para reflexões (resumo). Rev Bras Epidemiol 2002; Sup Especial: 279.

22. Sanches K. Características epidemiológicas dos casos de aids em idosos no estado do Rio de Janeiro (resumo). Rev Bras Epidemiol 2002; Sup Especial: 308.

23. Brito AM. A epidemia da aids em Pernambuco: sobrevida dos doentes no período de 1983 a 1995 [dissertação de mestrado]. Recife: Universidade Federal de Pernambuco; 1997.

24. Castilho E, Chequer P. A epidemia da aids no Brasil. In: Brasil. Ministério da Saúde. Coordenação Nacional de DST/aids. Simpósio satélite: A epidemia da aids no Brasil: Situação e tendências. Brasília, 1997.

25. Rouquayrol MZ, Almeida Filho N. A aids no Brasil. In: Rouquayrol MZ, Almeida Filho N. Epidemiologia e saúde. Rio de Janeiro: MEDSI; 1999. P. 271-84.

26. Sousa MGG. Geografia da aids em Pernambuco e desenvolvimento humano: 1983-1998 [monografia de especialização em epidemiologia]. Recife: Faculdade de Ciências Médicas da UPE; 1999.

27. Pernambuco. Aids: situação atual. Boletim Informativo DST/aids 2002; 2(1): 2-4.

28. Fonseca MGP, Szwarcwald CL, Derrico M, Andrade CLT, Veras CT, Bastos FI. Aids e grau de escolaridade no Brasil: evolução temporal de 1986 a 1996. Cad Saúde Pública 2000; 16: 77-87.

29. Bastos FI, Szwarcwald CL. Aids e pauperização: principais conceitos e evidências empíricas. Cad Saúde Pública 2000; 16: 65-76.

30. Ferro S, Salit IE. HIV infections in patients over 55 years of age. J Acquir Immune Defic Syndr Hum Retrovirol 1992; 05: 348-53.

31. Butt AA, Dascomb KK, Desalvo KB, Bazzano L, Kissinger PJ, Szerlip HM. Human immunodeficiency virus infection in elderly patients. South Med J2001; 4: 397-400.

Recebido em: 07/06/06 Versão final reapresentada em: 09/07/07 Aprovado em: 09/07/07 